\title{
EchoGéo
}

6 | 2008

La Guyane

\section{Coopération internationale locale et politique éducative en Guyane}

Isabelle Hidair et Pierre-Yves Chicot

\section{OpenEdition}

Journals

Édition électronique

URL : https://journals.openedition.org/echogeo/6753

DOI : $10.4000 /$ echogeo.6753

ISSN : 1963-1197

Éditeur

Pôle de recherche pour l'organisation et la diffusion de l'information géographique (CNRS UMR 8586)

Référence électronique

Isabelle Hidair et Pierre-Yves Chicot, "Coopération internationale locale et politique éducative en

Guyane », EchoGéo [En ligne], 6 | 2008, mis en ligne le 08 octobre 2008, consulté le 01 août 2021. URL : http://journals.openedition.org/echogeo/6753 ; DOI : https://doi.org/10.4000/echogeo.6753

Ce document a été généré automatiquement le 1 août 2021

EchoGéo est mis à disposition selon les termes de la licence Creative Commons Attribution - Pas d'Utilisation Commerciale - Pas de Modification 4.0 International (CC BY-NC-ND) 


\title{
Coopération internationale locale et politique éducative en Guyane
}

\author{
Isabelle Hidair et Pierre-Yves Chicot
}

1 Faut-il parler des compétences internationales locales, des compétences externes des communes, des départements et des régions françaises? La réponse ne fait pas l'objet d'une affirmation tranchée même si le débat a bien évolué depuis vingt ans. On peut considérer que depuis la légalisation de la coopération internationale décentralisée les collectivités territoriales sont titulaires d'une compétence internationale au même titre que l'État (Chicot, 2005). La base textuelle est le titre IV de la loi d'orientation n'92-125 dite ATR (loi d'orientation sur l'administration territoriale de la République) du 6 février $1992^{1}$. Cette position était déjà du reste défendue par une toute petite partie de la doctrine avant même la loi d'orientation du 6 février 1992. Le professeur François Luchaire (1983) tire cette conclusion à la lumière de la lettre de l'article 9 de la loi du 31 décembre 1982 et celle de l'article 13 de la loi du 2 août 1984 (op.cit.: 120-127).

2 Pour s'en tenir à la loi ATR, son article 131-I, codifié dans le code général des collectivités territoriales ${ }^{2}$, autorise l'établissement de rapports juridiques, par le biais de conventions, entre collectivités territoriales françaises et collectivités étrangères. Les dispositions qui suivront iront plus loin. C'est ainsi qu'il sera permis à une collectivité territoriale d'être placée, à l'occasion de rapports internationaux, sous l'emprise d'un droit étranger. La reconnaissance par le droit positif de la compétence internationale locale est finalement la traduction de la reconnaissance par le législateur de la localisation de l'intérêt local hors de la sphère juridique interne. La célèbre notion d'affaires locales ne vaut donc pas uniquement pour l'ordre juridique interne.

3 Cette question de la compétence internationale locale est d'autant plus intéressante qu'elle invite à une profonde réflexion sur l'État. Est-ce à dire qu'en matière d'affaires étrangères les collectivités secondaires de la République jouent dans la même cour que l'État? La réponse aura évidemment une valeur négative, et bien entendu les démembrements de la République ne sont pas sujets du droit international (Ndiaye, 1994 : 521). 
4 La police, la justice, la défense, la monnaie, les affaires étrangères, l'éducation nationale sont généralement citées comme faisant partie des compétences régaliennes qui ne sauraient être partagées. Pour autant, nombre d'observateurs de la doctrine du droit public français s'accordent pour dire que le modèle étatique français est rongé par le haut par la construction communautaire et par le bas par la décentralisation. Il est à l'évidence extrêmement difficile de contester aujourd'hui un tel état de fait, sauf à revenir de manière très véloce à l'État centralisé omnipotent et omniprésent qui irradiait les provinces de France hexagonale et de la France ultramarine de ses politiques publiques, de ses mesures et de ses normes décidées d'en haut.

5 Ce schéma est dépassé. L'acte II de la décentralisation dont le contenu normatif se trouve dans la loi constitutionnelle $\mathrm{n}^{\circ}$ 2003-276 du 28 mars $2003^{3}$ relative à l'organisation décentralisée de la République ainsi que dans la loi sur les libertés et les responsabilités locales ${ }^{4}$ restera comme l'une des très grandes réformes de la Vème République. La réforme constitutionnelle modifie considérablement la donne. Elle offre une consistance jamais égalée au droit constitutionnel local. À titre d'exemple parlant, l'inscription du principe de subsidiarité- consubstantiel aux États fédéraux- dans la norme fondamentale française ${ }^{5}$ offre un champ du possible extrêmement prometteur aux collectivités territoriales. La politique éducative pour le cas qui nous occupe en est un exemple patent. À l'instar d'autres domaines, la coopération internationale locale dans la politique éducative est présidée par le critère de la géographie qui met à nouveau en exergue le concept de l'altérité de proximité.

\section{Une pratique internationale présidée par le critère de la géographie}

6 En appelant à la rescousse du droit, pour une part minimale certes, la géographie, l'histoire et la sociologie, on en vient à conclure que le fondement socio-historique de la compétence internationale locale, à tout le moins pour ce qui concerne les départements-régions d'outre-mer réside dans l'altérité de proximité (Chicot, 2005 : 162). Les dispositions législatives relatives à la coopération internationale des départements-régions d'outre-mer constituent d'une part, l'antithèse du principe de l'exclusif qui commandait aux collectivités ultramarines d'entretenir des relations avec la seule métropole, et d'autre part, le produit de revendications de décideurs publics locaux relatives à la reconnaissance de la légitimité de l'action internationale locale.

7 En somme, la contestation du principe de l'exclusif, la volonté exprimée de pouvoir nouer des relations avec des collectivités territoriales, situées dans la même zone géographique résultent de la recherche d'une autre altérité : une altérité de proximité présidée par la géographie. Pour la Guyane, cette altérité de proximité présidée par la géographie prend essentiellement deux formes les relations de voisinage ainsi que la coopération formalisée ou à formaliser avec des organisations internationales à vocation régionale.

8 Située sur le plateau des Guyanes ${ }^{6}$, la Guyane française dispose de frontières communes avec deux États indépendants. À l'Orient, l'État fédéré d'Amapá de la République Fédérative du Brésil, à l'Occident, le Surinam dont la ville la plus proche est Albina, frontalière de la ville de Saint-Laurent-du-Maroni. 
9 La coopération internationale de la Guyane française va au-delà de la simple coopération transfrontalière. Il est par conséquent plus opportun d'utiliser l'expression « relations de voisinage » qui exprime les relations entretenues avec les collectivités du sous-continent, au Sud, et au Nord, avec les collectivités des États de la mer des Caraibes. Néanmoins à l'épreuve de la pratique, on observe que ces relations de voisinage sont essentiellement entretenues avec les États fédérés du Brésil.

10 Ces relations de voisinage sont à la fois horizontales et verticales. On va parler de relations horizontales, quand il s'agit d'aborder la coopération transfrontalière entre les collectivités territoriales de Guyane française avec leurs homologues étrangers du Brésil et du Surinam. Les relations verticales, pour leur part, sont celles effectives ou à venir avec des organisations internationales à vocation régionale.

\section{Les relations horizontales et collectivités territoriales du Brésil}

11 Les relations internationales nourries entre la Guyane française et les collectivités fédérées du Brésil reposent sur un accord cadre de coopération entre la France et le Brésil conclu le 28 mai 1996. Depuis cet accord inter-étatique qui n'est pas pour autant un « umbrella agreement $»^{7}$, les relations inter-institutionnelles entre la Guyane française et la collectivité fédérée d'Amapá se sont considérablement développées ${ }^{8}$. Au-delà du contenu classique ${ }^{9}$ de cette coopération décentralisée, il faut insister sur les motivations politiques des pouvoirs locaux brésiliens et guyanais. Les deux collectivités infraétatiques invoquent leur position périphérique et ultrapériphérique des centres décisionnels brésiliens et franco-communautaires pour dynamiser les potentialités offertes par la proximité frontalière. Dans ce cas d'espèce, le concept de l'altérité proximité est immanquablement invocable.

12 En revanche, les relations qui se mettent en place entre la Guyane française d'une part, et l'État du Pará ${ }^{10}$ et d'Amazonas ${ }^{11}$ d'autre part, sont essentiellement motivées par des arguments économiques et commerciaux. La coopération internationale décentralisée franco-brésilienne est aussi l'œuvre des principales villes de ce département-région ${ }^{12}$ français d'Amérique du Sud. C'est ainsi que Cayenne et Saint-Laurent-du-Maroni ${ }^{13}$ entretiennent des liens internationaux avec des homologues étrangers de leur zone géographique. La ville de Cayenne, qui développe «le concept de ville-capitale ${ }^{14}$, a signé en décembre 2005 un accord international infraétatique avec la ville de Salvador, capitale de l'État fédéré de Bahia au Brésil. La ville de Saint-Laurent-du-Maroni qui ne dispose pas d'une frontière terrestre avec la ville d'Albina ${ }^{15}$ en raison de la présence de l'imposant fleuve Maroni n'en est pas moins très proche.

13 Ce sont principalement les échanges naturels des populations résidant des deux côtés $\mathrm{du}$ fleuve qui ont conduit les deux collectivités municipales à formaliser des relations institutionnelles. Au-delà des problèmes sociaux posés, la proximité de Saint-Laurentdu-Maroni avec les deux autres Guyanes contient de potentiels bénéfices qui peuvent être profitables à la commune. La stabilité que connaît le Surinam depuis la fin de la guerre civile lui a permis d'engager des discussions en vue d'une coopération " forte et rapide $»^{16}$ avec la Guyane française. La ville de Saint-Laurent-du-Maroni représente donc un acteur public incontournable de cette politique internationale d'ensemble du département-région Guyane. La ville de Saint-Laurent du Maroni apparaît comme le 
point d'appui naturel de la politique de coopération régionale de la Guyane en direction du Surinam et du Guyana.

Ces relations horizontales mettent en exergue l'autonomie politique dont font preuve les autorités territoriales. En dépit, de l'existence des fonds de coopération régionale (FCR) gérés par la préfecture de la Guyane ${ }^{17}$, les collectivités territoriales préfèrent pour ne pas faire l'objet d'un double contrôle ${ }^{18}$ affecter des ressources financières locales à leurs politiques locales internationales. C'est tout simplement la volonté d'éviter l'immixtion de l'État dans les politiques publiques internationales d'essence locale qui explique cette stratégie financière. Par contre, on observe une envie tenace des autorités territoriales d'empiéter sur les plates-bandes de l'État. C'est ainsi qu'apparaissent des relations verticales. Autrement dit, entre des sujets de droit international et des entités juridiques qui ne le sont pas.

\section{Les relations verticales et relations diplomatiques de l'État}

Si la coopération internationale décentralisée s'est développée à la faveur des concessions du pouvoir central, ce dernier veille scrupuleusement à ce que l'ordre des pouvoirs au sein de la République soit respecté. L'action extérieure a longtemps été l'apanage exclusif de l'État. Sur le plan de l'histoire, l'apparition récente des politiques publiques internationales locales, exception faite du jumelage municipal ${ }^{19}$, peut être présentée comme une compétence arrogée qui a finalement été prise en compte par la loi, en 1982 et en $1992^{20}$.

Dans les départements-régions français d'Amérique, la coopération internationale décentralisée est parfois synonyme d'une défiance vis-à-vis de l'État. On ne peut donc pas totalement exclure des relents de sédition de la part de certains exécutifs locaux. Le législateur en affirmant de manière péremptoire que toute relation entre une collectivité territoriale et un État est interdite, rappelle le rôle prééminent de l'État dans les relations extérieures de la France ${ }^{21}$. Il indique en outre, que si la coopération internationale donne lieu à une concurrence dans l'action entre l'État et les collectivités territoriales, il n'en est rien pour ce qui concerne les affaires diplomatiques. Ce dernier domaine ne peut donc être partagé avec les collectivités secondaires.

17 Pourtant, de nouvelles exceptions vont être permises pour les départements régions d'outre-mer ce qui justifie bien l'existence d'un régime juridique différencié de la coopération internationale décentralisée dès lors qu'on est en présence des collectivités ultramarines de droit commun. Ce régime juridique différencié de la coopération internationale décentralisée va jusqu'à la consécration d'un début de décentralisation diplomatique en droit français au bénéfice des collectivités ultramarines de droit commun. C'est l'interprétation qu'on peut déduire des articles 42 et 43 de la loi d'orientation $\mathrm{n}^{\circ}$ 2000-1207 pour l'outre-mer du 13 décembre 2000. Votée dans le contexte d'une réclamation bruyante de l'abolition du statut départemental ${ }^{22}$, le législateur organise le cadre juridique d'une évolution institutionnelle dans les départements-régions d'outre-mer par la légalisation du congrès. A l'occasion de cette production législative, le législateur aligne également la compétence internationale de ces collectivités sur celle exercée par les anciens territoires d'outre-mer de l'article 74. 
Concrètement, les exécutifs départementaux et régionaux peuvent adresser au Gouvernement des propositions de traités à signer avec des États de leur zone géographique, avec des organisations internationales de la région y compris avec des institutions spécialisées de l'ONU. Le Gouvernement peut aussi autoriser les exécutifs locaux à négocier des accords avec un État, un territoire ou une organisation internationale. Enfin, les exécutifs départementaux et régionaux peuvent représenter la France au sein d'instances internationales et parler en son nom.

Quel est le bilan? L'État délivre avec la plus grande parcimonie des autorisations d'immixtion dans le champ diplomatique. La logique est respectée puisque l'essor de l'action internationale locale reste pour une large part étroitement dépendant des concessions étatiques. Par contre, curieusement, on ne peut pas dire que les collectivités territoriales françaises d'Amérique, et en l'occurrence celles de Guyane française usent des moyens offerts par la loi. C'est ainsi que la situation sociale de la Guyane enjoint les autorités locales et les autorités déconcentrées tel que le rectorat à développer des actions de coopération transfrontalière de proximité. À cet égard un véritable diagnostic territorial, sociologique est nécessaire, préalable à la l'élaboration des actions de coopération en matière de politique éducative.

\section{La Guyane française : la natalité la plus importante de France}

20 La Guyane française est le département français d'Amérique qui connaît la croissance démographique la plus forte, il est le plus étendu et le moins peuplé de l'Outre-mer. Ainsi, en tant que département français et territoire européen sur le continent sudaméricain, la Guyane présente une originalité géopolitique majeure et attire les ressortissants des pays voisins chassés par les guerres civiles, les dictatures, la récession économique, le chômage et les raisons de santé.

En 2004, la population guyanaise est estimée à 178347 habitants et en 2002, la population clandestine, dont il faut souligner les difficultés d'évaluation statistique, est estimée à 30000 personnes selon la Préfecture. Lors d'une étude réalisée en 1997, le Conseil Économique et Social Régional a estimé la population guyanaise à 300000 individus en 2015 soit un doublement en vingt ans (Hidair, 2003 : 87). Ainsi, selon l'INSEE, en 2030, la population de la Guyane devrait compter 42,5\% de personnes de moins de 20 ans, $46,7 \%$ de personnes entre 20 et 59 ans et $10,9 \%$ de personnes de 60 ans et plus, se situant au total aux environs de 400000 personnes. Le taux de natalité est de $14 \%$ en France, de $38 \%$ en Guyane et plus particulièrement sur le fleuve Maroni où il est de $45 \%_{0}{ }^{23}$. Soulignons que cette explosion démographique n'est pas attribuée au seul solde naturel mais aussi au solde migratoire. Depuis, 1990, le constat est fait que plus de la moitié des naissances proviennent de femmes étrangères. En 2003, cette proportion s'élève à $58,8 \%$ des naissances annuelles. "Les familles dont le chef de ménage est étranger représentent près de $30 \%$ de l'ensemble des familles guyanaises. Celles-ci sont plus souvent des familles nombreuses que les familles dont le chef de ménage est Français. Près de $40 \%$ d'entre elles ont plus de 3 enfants, contre $20 \%$ pour les familles françaises" (Charrier, 2002a : 20). En 1999, les femmes de Guyane mettent au monde durant leur vie 3,9 enfants. Elles détiennent presque le record d'Amérique du sud et des Caraïbes. Suivant leur nationalité, les femmes n'ont pas le même nombre d'enfants. En effet, l'étude de l'NSEE nous apprend que les femmes Surinamaises ${ }^{24}$, les Créoles 
haïtiennes ${ }^{25}$ et les Brésiliennes ont un comportement nataliste assez proche des pays sous développés alors que le comportement démographique des familles françaises en Guyane a tendance à se rapprocher de celui de la France métropolitaine. Ainsi, les habitants de Guyane de "ces trois principales communautés étrangères contribuent pour près de $50 \%$ au solde naturel. Ces populations se caractérisent par une très faible part de personnes âgées ${ }^{26}$ donc une mortalité faible » (Charrier, 2002b : 16).

Cependant, certains bébés comptés naissent de femmes qui, elles, ne sont pas comptées. En effet, un certain nombre de Surinamaises traversent le Maroni afin d'accoucher en Guyane. À Saint-Laurent-du-Maroni, on estime l'indicateur conjoncturel de fécondité des Surinamaises à plus de 7,5. Ce chiffre est disproportionné en comparaison de celui du Surinam (entre 2,5 et 3 enfants par femme en 2001).

«Si en 1990, les immigrés étaient surtout des hommes (55\%) venus travailler sur les grands chantiers de Guyane, la tendance s'est renversée dix ans plus tard puisque les femmes deviennent majoritaires. En 1992 et 1993, l'Office des migrations internationales (OMI actuelle $\mathrm{ANAEM}^{27}$ ) a procédé à une grande opération de régularisation provoquant une hausse des demandes de regroupement familial, notamment pour les familles haïtiennes. La proportion de femmes immigrées en âge de procréer s'est donc accrue "(Charrier, 2002b: 16). L'industrie, le bâtiment, et l'agriculture restent, pour la majorité de leurs ressortissants Surinamais, Haïtiens et Brésiliens, les activités professionnelles de prédilection.

À la lecture du tableau suivant, les constatations qui s'imposent sont que, d'une part, ces données ne tiennent pas compte de l'immigration clandestine et sous-évaluent les nouvelles implantations noires marronnes ${ }^{28}$ de l'Ouest guyanais (Hidair, 2003).

Structure de la population étrangère en Guyane de 1990 à 1999

\begin{tabular}{|l|c|c|c|c|}
\hline Nationalités & 1990 & $\begin{array}{c}\text { Pourcentage de } \\
\text { la population } \\
\text { totale } \\
\text { en } 1990\end{array}$ & 1999 & $\begin{array}{c}\text { Pourcentage de } \\
\text { la population } \\
\text { totale } \\
\text { en } 1999\end{array}$ \\
\hline Surinam & 13296 & 11,58 & 17654 & 11,26 \\
Haïti & 8899 & 7,75 & 14143 & 9,02 \\
Brésil & 5615 & 4,89 & 7171 & 4,57 \\
Guyana & 1648 & 1,43 & 2372 & 1,51 \\
Union européenne & 1195 & 1,04 & 846 & 0,53 \\
Sainte-Lucie & 835 & 0,77 & 525 & 0,33 \\
République Dominicaine & 392 & 0,34 & 673 & 0,42 \\
Autres nationalités & 2122 & 1,85 & 3192 & 2,03 \\
Total de la population étrangère & 34002 & 29,65 & 46576 & 29,67 \\
\hline
\end{tabular}

Source : Montoute et Nancy, 1995 : 17 et Recensement 1999

25 Aujourd'hui, la population guyanaise est composée des groupes socioculturels suivants : Africains, Amérindiens, Brésiliens, Chinois, Créoles antillais, guyanais, réunionnais, haïtiens et sainte-luciens, Guyaniens, Dominicains, Hmong, Libanais, Métropolitains, Noirs marrons ${ }^{29}$, Péruviens, Surinamais, Vénézuéliens.... Cette liste non exhaustive permet de comprendre que, même si la culture créole domine pour le moment, il se pose le problème de l'équilibre entre les communautés dans une société multiculturelle où les complexités socioéconomiques émergent (Hidair, 2003). 


\section{Un accueil scolaire de plus en plus difficile} et $33 \%$ des élèves de $3^{\mathrm{e}}$ ont un retard de deux ans et plus (Rectorat/INSEE) ${ }^{31}$. Les résultats de l'académie de la Guyane à la session 2004 du baccalauréat, tous candidats confondus, reste le plus bas des DOM et de la France métropolitaine. Les disparités sont particulièrement observées lorsque les enfants sont issus de ménages non francophones. Dans ce contexte, le risque est de développer une éducation à deux vitesses entre le public et le privé, se différenciant par la classe sociale et l'appartenance culturelle. Selon le Conseil Général, « le manque de diplômés, le retard scolaire, la faiblesse du retour au pays des jeunes diplômés (13\%), parmi lesquels figurent aussi les immigrés qui ont bénéficié d'une scolarisation en primaire et en secondaire en Guyane donne à penser qu'il faudra encore plusieurs générations pour produire la totalité des cadres dont elle a besoin, voire répondre équitablement aux appels d'offre nationaux en matière de postes d'encadrement » (INSEE-Conseil général, 2005). En s'appuyant sur ces réalités, le rectorat de la Guyane a décidé d'œuvrer pour le développement des relations de coopération avec le Surinam.

31 Les problèmes de non scolarisation et de déscolarisation sont les plus nombreux dans le bassin de l'Ouest. Plusieurs facteurs contribuent aux difficultés de scolarisation rencontrées : implantation géographique des écoles existantes, manque d'infrastructures scolaires, nécessité du développement d'un enseignement secondaire, équipements insuffisants, problèmes de transports scolaires, écarts linguisticoculturels importants entre les enfants et l'école, dévalorisation de ces zones par les 
enseignants (manque de logements, difficulté de déplacements, manque de formation) et manque de qualification des enseignants en poste.

\section{Une approche concertée de la coopération éducative}

Différents groupes socioculturels peuplent les rives du Maroni, mais les plus nombreux à émigrer vers la Guyane sont les descendants d'esclaves marrons.

Les quatre principaux groupes rencontrés sont les Aluku, les Ndyuka, les Paramaka et les Saramaka ${ }^{32}$. On compte aujourd'hui 37200 Marrons en Guyane (Price, 2003: 69). En représentant $20 \%$ de la population guyanaise, les Marrons «sont donc, après les Créoles, plus forts en nombre que toute autre composante de la population guyanaise " (ibid.). Ces quatre groupes «se sont formés en Guyane hollandaise (Surinam) aux XVII et XVIII ${ }^{e}$ siècles. «Vers 1970, la Guyane ne [compte] que 7500 Marrons environ - 3000 Saramaka (des hommes qui y [travaillent] pour une durée de quelques années) et 2000 Aluku (la quasi-totalité de la population aluku, qui [a] déjà la nationalité française) ainsi que 2000 Ndyuka et 500 Paramaka qui [ont] des abattis sur la rive française ou [ont] migré à Saint-Laurent et ses environs. La grande majorité de ces Marrons se [trouve] sur le Maroni ou le Lawa et à Kourou » (op.cit. : 69).

En 1986, une guerre civile éclate à la suite d'un différend personnel entre le dirigeant du Surinam, le Commandant Desie Bouterse, et Ndjuka Ronnie Brunsjwick (Bougarel, 1990 : 43), sergent de l'armée surinamienne et garde du corps de Bouterse. Après un massacre de civils, l'exode de la population commence. À ce moment, la population de Saint-Laurent-du-Maroni se multiplie par deux et des migrants se fondent dans la population noire marronne de la région, accueillie par des parents ou amis vivant depuis plusieurs années en Guyane (Reginensi, 1996 : 41-42). Des camps sont installés, mais le statut de réfugiés politiques leur est refusé par la France. Ils sont considérés comme des PPDS : «Personnes Provisoirement Déplacées du Surinam ». En 1992, une aide au retour est accordée par le ministère des DOM-TOM, mais seuls 600 réfugiés (sur un total de 6500 ) se laissent convaincre, d'autres s'enfuient dans la forêt ou trouvent un hébergement (ibid.). Plus tard, certains « ont acquis une carte de séjour ; quelquesuns, plus rares, la nationalité française et vivent dans des situations de précarité (RMI, petits boulots...) » (ibid.). Le géographe Frédéric Piantoni [2002 (2006)] rappelle que « les six années d'affrontement, de 1986 à 1992, marquent une rupture radicale dans l'organisation démo-spatiale du fleuve, en débouchant sur la matérialisation d'une région fluviale et transfrontalière ». Actuellement, des familles installées sur les deux rives se voient séparées par leurs nationalités étrangères alors que les alliances et les échanges économiques existent depuis les débuts de la colonisation (Piantoni, [2002 (2006)]. L'économie transfrontalière tire sa source de l'activité aurifère et est perceptible par l'émergence de «kampus (hameaux-campements). Ils se structurent autour d'une épicerie, souvent d'une petite boîte de nuit, d'une pyramide d'une cinquantaine de fûts métalliques de 200 litres qui tient lieu de station d'essence et de réserves de carburant destinées au fonctionnement des groupes électrogènes et des machines d'extraction. (...) On peut donc établir une distinction entre les acteurs de l'économie informelle. On trouve d'abord ceux pour qui l'activité informelle est une stratégie de survie et dont le cadre d'action se limite à une aire d'action estuarienne sans possibilités de s'en éloigner en l'absence de régularisation administrative. Pour les autres, ceux ayant une situation professionnelle dans la société nationale, ils sont 
capables de constituer des réseaux d'échange importants grâce à leur couverture légale. Leur rayon d'action est généralement intégré à la totalité des territoires guyanais et surinamais et leur présence dans le département est généralement ancienne, datant des années 1960-1970» (ibid.). Ainsi, les liens historiques, géographiques, socioéconomiques et culturels unissant les deux rives du Maroni ne peuvent plus être niés et s'inscrivent désormais dans le cadre d'une politique éducative. La coopération éducative, menée depuis septembre 2005, consiste à mener des actions communes entre les états frontaliers en confrontant les donnéesstatistiques et démographiques, en harmonisant les constructions de part et d'autre du fleuve, en identifiant les circuits financiers habituellement sollicités sur chaque territoire et en définissant une nouvelle approche pédagogique adaptée aux populations concernées. Les objectifs sont visent donc à l'amélioration de la programmation des constructions, l'atténuation des disparités en proposant le même enseignement des deux côtés des fleuves, la fixation des populations en scolarisant les enfants sur leur lieu de vie, le rééquilibrage des frontières par la pédagogie (notamment l'enseignement du français et du néerlandais), mais aussi par l'économie en soutenant les productions locales (ouvrages, matériels, cantine). De plus, la création d'un article de $\operatorname{loi}^{33}$, proposant un statut spécifique aux enseignants dispensant des cours dans les établissements appartenant au dispositif, faciliterait les déplacements de ces derniers en vue de dynamiser les échanges pédagogiques. Ce statut particulier serait accordé uniquement aux enseignants exerçant à la fois dans les écoles surinamaises et françaises.

À la suite de nombreuses rencontres avec les partenaires institutionnels du Surinam, trois projets ont vu le jour. Tout d'abord, la construction d'une classe sur la rive surinamaise a été réalisée. Puis, deux assistants néerlandophones ont été recrutés afin d'intervenir dans les classes françaises. Ceci est la première étape qui permet l'intervention d'assistants de langue des deux côtés de la frontière. Enfin, l'ouverture d'une antenne de l'Institut de Formation des Maitres au Surinam favorisera l'installation de professeurs des écoles entre les deux régions. Cependant, force est de constater que la multiculturalité et le multilinguisme ${ }^{34}$ guyanais et surinamais ne sont toujours pas pris en compte par le rectorat de Guyane. En effet, les deux langues de communications choisies dans les écoles participant au projet, à savoir le français et le néerlandais, ne sont pas les langues maternelles de la majorité des enfants scolarisés dans cette zone. Sur ce point, la linguiste Isabelle Léglise souligne que «hormis l'émerillon, toutes les langues amérindiennes parlées en Guyane sont transfrontalières (Goury et al., 2000), en particulier le kali'na (parlé également au Brésil, au Surinam, au Guyana et au Vénézuéla), et le palikur (parlé au Brésil). De même, les variantes nenge, issues du marronnage d'esclaves venant des plantations du Surinam au XVIII ${ }^{\mathrm{e}}$ siècle, sont actuellement parlées de part et d'autre du fleuve Maroni » (Léglise, 2004). 
En Guyane: " outre le français, langue officielle, on compte une dizaine de langues ayant les caractéristiques de citoyenneté et territorialité des langues régionales (Launey, 1999, Camargo et al., 2003) : le créole guyanais, des langues amérindiennes (kali'na, wayana, wayampi, émerillon, arawak et palikur), des créoles de base lexicale anglaise (ou nenge sous trois variantes dialectales : aluku, ndjuka et pamaka) et de base anglaise relexifiée partiellement en portugais (le saamaka) et enfin, depuis les années 70, une langue asiatique, le hmong » (Léglise 2004).

Au Surinam : «le profil linguistique des personnes originaires du Surinam ne peut se deviner à partir de la nationalité de ces dernières, car outre le néerlandais ou hollandais, un certain nombre de langues y co-existent: le sranan tongo (créole servant de véhiculaire au Surinam dans les communications inter-communautaires (Carlin, 2001) et langue première d'un certain nombre de locuteurs, en particulier les Créoles surinamais), les langues des marrons (ndjuka, aluku, pamaka, kwinti, saamaka...), des langues amérindiennes (kali’na, arawak, wayana, tirio...), des langues provenant de premières migrations (créole anglais du Guyana, javanais, hindoustani, chinois hakka...). La majorité des habitants du Surinam est ainsi locutrice de langues minoritaires auxquelles s'ajoutent parfois le néerlandais et le sranan tongo, a fortiori s'il s'agit d'une population vivant en zone urbaine et scolarisée » (Léglise, 2004).

De plus, l'auteur met en évidence les bons taux de transmission de la première langue qui est fortement valorisée. "La langue des ancêtres est en effet vécue comme moyen identitaire fort dans les communautés businenges locutrices d'aluku, ndjuka ou pamaka (Léglise et Migge, 2003). Toutefois, le statut de ces langues dans la société globale, au Surinam comme en Guyane, n'est pas élevé : d'après R. Price et S. Price (2003) ces langues se situent tout en bas de l'échelle sociale" (ibid.). Ce dernier constat est confirmé par la démarche du rectorat de la Guyane qui ne prend pas en compte la diversité des aspects sociolinguistiques et culturels qui permettraient de mener à bien cette initiative de coopération transfrontalière.

Complètement immergés dans l'univers des affaires internationales en raison de changements de circonstances liés aux transformations mêmes du monde, les départements-régions français d'Amérique sont de facto et de jure, titulaires de la compétence internationale. De jure, ces collectivités sont investies de la «capacité de faire ", de facto, on peut légitimement s'interroger à certains moments sur la " capacité à faire ». Les vastes chantiers de la coopération internationale qui s'ouvrent pour la Guadeloupe, la Guyane et la Martinique, mais également pour la Réunion- bien que située dans une autre zone géographique- doivent les inciter à la sagesse et à la prudence en matière de revendication de compétences qui supposent moyens financiers, matériels et humains supplémentaires. En revanche, la nécessité de renforcer leur capacité d'expertise au sein des administrations locales et déconcentrées se révèlent être une nécessité indiscutable. La politique éducative apparaît comme un exemple illustratif puisque l'État et les collectivités territoriales disposent de compétences complémentaires dans ce domaine.

Nonobstant la montée en puissance des départements-régions d'outre-mer dans l'ordre juridique international, l'État conserve toute sa place et reste incontestablement l'acteur premier. La compétence internationale locale en droit français « ne porte pas atteinte à l'ordre des pouvoirs dans la République » selon la célèbre formule du Conseil d'État utilisée par exemple dans son avis du 16 décembre 1980 concernant la signature par la France de la convention-cadre de Madrid sur la coopération transfrontalière (Decaux, 1984 : 557-620).En fait, malgré les mutations subies par l'État français qui vont inéluctablement dans le sens d'un affadissement du jacobinisme, ces mutations respectent un équilibre exprimé de fort belle manière par Paul Valéry: «si l'État est fort il nous écrase, s'il est faible nous périssons ». 


\section{Lexique}

ANAEM : Agence Nationale d'accueil des étrangers et des migrations.

ATR : loi d'orientation sur l'administration territoriale de la République.

FCR : fonds de coopération régionale.

OMI (actuelle ANAEM) : Office des migrations internationales

\section{BIBLIOGRAPHIE}

Archives nationales section outre-mer, séance du 10 juin 1861. Guyane, Carton 55 F3 (09) Conseil privé.

Antiane éco, 1991. INSEE, nº15, p.14 à 18.

Bougarel, S. 1990. «Les réfugiés surinamiens en Guyane », Études Créoles. Cultures, langue, société. Numéro spécial Guyane, Montréal : Didier Érudition, Volume XIII, n², pp. 43-49.

Chambre de commerce et d'industrie de la Guyane, 1999. Quelle politique de développement adaptée à la coopération régionale?, Journées-pays : Surinam, 31 mars-01 avril, Synthèse des ateliers, p.13.

Charrier, R. 2002a. « La famille guyanaise nombreuse », AntianeÉco n 51, p. 19-21.

Charrier, R. 2002b. « Guyane, des peuples et des histoires », AntianeÉco n 54, p. 14-17.

Chicot, P-Y. 2005. La compétence internationale des collectivités territoriales françaises : l'action extérieure des départements des Antilles et de la Guyane, Paris : L'Harmattan.

Conseil Économique et Social Régional (CESR), février 1997. Tourisme facteur de développement de l'Outre-mer français.

Conseil Général, Direction de la coopération, mars 2005. Impact de la croissance démographique sur les actions du Conseil Général : le poids de l'immigration, Rapport à Monsieur le Ministre Michel Barnier.

Conseil Régional, Conseil Général, Préfecture de la Guyane, Conseil Économique et Social Régional, 1997. Plan de développement Régional.

Decaux, E. 1984. La convention cadre européenne sur la coopération transfrontalière des collectivités ou autorités territoriales, RGDIP, p. 557-620.

Hidair, I. 2003. La construction identitaire des Créoles guyanais de Cayenne. De la gestion d'un héritage pluriel à l'insertion dans un contexte multiculturel, Paris : Thèse de doctorat, EHESS.

Léglise, I. 2004. « Frontières de langues et langues de frontières », dans ML. Moreau (coord), Glottopol n 4, Université de Rouen, p. 108-124.

Luchaire, F. 1983. La décentralisation dans les départements d'outre-mer, AJDA, p.20-127.

Montoute, D. et Nancy, L. 1995. Femmes de Guyane, Cayenne : INSEE.

Ndiaye, P. 1994, Les collectivités territoriales et l'organisation internationale : état et perspectives de la centralisation des relations internationales dans le cadre unitaire français, Montpellier I, $521 \mathrm{p}$. 
Piantoni,F.2006 [2002]. « Les recompositions territoriales dans le Maroni : relation mobilitéenvironnement », Revue Européenne des Migrations Internationales, Volume 18, Numéro 2, p. 11-49, http://remi.revues.org/document1630.html

Price, R. \& Price, S. 2003. Les Marrons, Châteauneuf-le-Rouge : Vents d'ailleurs.

Réginensi, C. 1996. Vouloir la ville. Du « business » à la citoyenneté en Guyane Française, Saint-Estève : Éditions de L'Espérou.

Tableaux Économiques Régionaux

1997. INSEE, Guadeloupe PRIM - ZI Jarry, 154p.

2000. INSEE, Guadeloupe PRIM - ZI Jarry, 158p.

2002. INSEE, Guadeloupe PRIM - ZI Jarry, 152p.

\section{NOTES}

1. Journal Officiel de la République Française (JORF), 8 février 1992, p. 2064.

2. Article L 1112-1.

3. JORF, 29 mars 2003, p. 5568.

4. Loi n ${ }^{\circ}$ 2004-809 du 13 août 2004, JORF, 17 août 2004, p. 14545.

5. Article 72 alinéa 2 de la Constitution du 4 Octobre 1958.

6. Le plateau des Guyanes est composé de: la Guyane française, du Surinam (ex Guyane hollandaise), de la République coopérative de Guyana (ex Guyane anglaise) et du Nord-Est du Brésil (l'État d'Amapá).

7. En effet, l'accord inter-étatique entre la France et le Brésil vise d'après l'article $1^{\text {er }}$ à donner un « nouvel élan à leurs relations bilatérales. À cette fin, elles sont résolues à favoriser les contacts politiques à tous les niveaux entre les deux États et à renforcer le développement de leur coopération économique, culturelle, scientifique et technique... ».

8. Suite à l'accord du 28 mai 1996, les présidents du conseil régional et du conseil général de la Guyane ainsi que le gouverneur de l'État d'Amapá ont signé le 11 juin 1996, à Macapá, en présence du préfet de région, une déclaration d'intention qui formalise les modalités de mise en place de relations internationales locales entre le conseil régional et le conseil général de la Guyane d'une part, et l'État fédéré d'Amapá, d'autre part.

9. Santé, environnement, développement économique, culture.

10. La ville de Belém connaît une expansion rapide. Le gouverneur de l'État du Pará, Monsieur Simao Jatén a rendu officiellement visite au président du conseil régional de la Guyane en vue d'instituer un processus de coopération entre les deux collectivités.

11. La foire internationale de Manaus est l'occasion pour les collectivités territoriales de Guyane de mettre en œuvre une démarche de marketing territorial.

12. La Guyane est une région monodépartementale.

13. Saint-Laurent-du-Maroni est la ville française frontalière au Suriname. Le fleuve Maroni est la frontière naturelle qui sépare la Guyane du Suriname.

14. Ce concept signifie que non seulement Cayenne est le chef-lieu (capitale) de la Guyane française mais elle doit aussi jouer un rôle de moteur dans le développement du départementrégion. C'est l'esprit de la démarche initiée en janvier 2006 par les maires des «villes-capitales » des trois départements régions français d'Amérique (Fort-de France, Basse-Terre, Pointe-à-Pitre, Cayenne). Ils entendent agir sur l'élaboration du cadre de référence stratégique national qui détermine l'orientation des fonds communautaires et ce, pour la période 2007-2013.

15. Albina est la ville surinamaise frontalière à la Guyane. 
16. Chambre de Commerce et d'Industrie de la Guyane, 31 mars-01 avril 1999, p.13.

17. Décret 2001-314 du 11 avril 2001 relatif à la coopération régionale des départements-régions d'outre-mer, JORF, 14 avril 2001, p. 5801.

18. Un contrôle en amont de l'action internationale en comité d'examen pour l'allocation des FCR et un contrôle de la préfecture, en aval, dans le cadre du contrôle de légalité.

19. L'autorisation juridique des jumelages date de la IVème République. C'est le décret du 24 janvier 1956 modifié par le décret du 23 juin 1957 qui l'institue.

20. L'article 65 de la loi $n^{\circ} 82-213$ du 2 mars 1982 relative aux libertés des communes, des départements et des régions. Les articles 131 et suivants de la loi d'orientation $n^{\circ}$ 92-125 sur l'administration territoriale de la République.

21. L'article 83 de la loi d'orientation $n^{\circ}$ 95-115 du 4 février 1995 sur l'aménagement et le développement du territoire, JORF, 5 février 1995.

22. La déclaration de Basse-Terre du $1^{\text {er }}$ décembre 1999 est le résultat d'une initiative des trois Présidents des régions françaises d'Amérique. Dans un texte intitulé : «le courage politique au service du développement $"$.

23. Recensement 1999, INSEE.

24. Aussi appelés Surinamiens, ce nom désigne les ressortissants du Surinam (ex Guyane hollandaise).

25. Ce nom désigne les Créoles originaires d'Haïti. Il est nécessaire de préciser «Créole » car il existe d'autres populations créoles en Guyane.

26. À peine $3 \%$ des Surinamais ont plus de 60 ans (Charrier, 2002b : 16).

27. Agence Nationale d'accueil des étrangers et des migrations.

28. La majorité des Surinamais installés en Guyane appartiennent aux groupes socioculturels des Noirs marrons.

29. Sont appelés Noirs marrons ou Businenge, les descendants d'esclaves marrons. Ils sont majoritairement originaires du Surinam.

30. Désigne le quart nord-ouest frontalier au Surinam.

31. Le taux de scolarisation des 16-29 ans, en 2003, était de $35 \%$ en Guyane, de $44 \%$ aux Antilles et de $75 \%$ en France métropolitaine.

32. Des variantes orthographiques existent pour ces noms.

33. Cette proposition a été faite à la Commission chargée d'apprécier les conditions d'immigration en Guyane à l'issue de la réunion de travail du 5 mai 2006.

34. Voir encadré.

\section{RÉSUMÉS}

En matière de coopération, l'évolution des enjeux, pris en compte dans les politiques communautaires, se heurte aux réalités de terrain. La relative facilité qu'il existe à imbriquer les territoires en Europe continentale ne va pas de soi pour l'environnement guyanais. En effet, la Guyane est soumise à une logique de double intégration : intégration à l'Europe et intégration dans sa zone géographique. Ainsi, en dépit de l'héritage historique et de la présence de la France et de l'Europe, la Guyane doit penser son développement à partir de sa zone géographique d'implantation. De ce fait, l'action de coopération, menée depuis septembre 2005, consiste à présenter une politique commune d'éducation entre les états frontaliers en confrontant les 
données statistiques et démographiques, en harmonisant les constructions de part et d'autre du fleuve frontière Maroni, en identifiant les circuits financiers habituellement sollicités sur chaque territoire et surtout en définissant une nouvelle approche pédagogique adaptée aux populations concernées.

Out of matter of co-operation, the evolution of the stakes, taken into account in the Community policies, runs up against realities of ground. The relative facility which there exists to imbricate the territories in continental Europe does not go from oneself for the Guianese environment.

Indeed, Guiana is subjected to a logic of double integration: integration in Europe and integration in its geographical area.Thus, in spite of the historical heritage and the presence of France and Europe, Guiana must think its development starting from its geographical area of establishment. So the co-operation project, carried out since September 2005, consists in presenting a common policy of education between the frontier states by confronting the statistical and demographic data, by harmonizing constructions on both sides of the river border Maroni, by identifying the financial circuits usually requested on each territory and especially by defining a new teaching approach adapted to the populations concerned.

En cuanto a cooperación, la evolución de lo que está en juego a niveles, tenidos en cuenta en las políticas comunitarias, se choca con las realidades de terreno. La relativa facilidad que existe a imbricar los territorios en Europa continental no va de sí para el medio ambiente de Guyana. En efecto, Guyana se somete a una lógica de doble integración: integración a Europa e integración en su zona geográfica. Así pues, a pesar de la herencia histórica y la presencia de Francia y Europa, Guyana debe pensar su desarrollo a partir de su zona geográfica de implantación. Por lo tanto, la medida de cooperación, llevado desde septiembre de 2005, consiste en presentar una política común de educación entre los Estados fronterizos enfrentando los datos estadísticos y demográficos, armonizando las construcciones por una y otra parte del río frontera Maroni, identificando los circuitos financieros habitualmente solicitados en cada territorio y sobre todo definiendo un nuevo enfoque pedagógico adaptado a las poblaciones interesadas.

INDEX

Mots-clés : coopération transfrontalière, frontière, Guyane française, politique éducative, Surinam

Keywords : border, educational policy, French Guiana, Surinam, transborder cooperation Palabras claves : Cooperación transfronteriza, Frontera, Guyana francesa, Política educativa, Surinam

\section{AUTEURS}

\section{ISABELLE HIDAIR}

Isabelle Hidair (ishidair@wanadoo.fr) est Docteur en anthropologie sociale et ethnologie, Chargée de cours à l'université des Antilles et de la Guyane ; elle a notamment publié : - 2008 « Exemple de la stigmatisation de l'immigration créole haïtienne dans une école cayennaise ", dans Diversité, Accueillir les élèves nouveaux arrivants, Cortier, Cl., (dir.) 153, Juin, INRP

- 2008. «L'immigration étrangère en Guyane : Entre stigmatisation et stratégie de récupération », dans L'observatoire de l'institutionnalisation de la xénophobie, Observ.i.x. Revue Asylon(s), $\mathrm{n}^{\circ} 4$, mai 2008, Sciences Humaines et Sociales - Exilés, migrations, asiles, solidarités, accueils, 
nationalismes, xénophobies [http://observix.lautre.net]

- 2008 (à paraître). « Déterminisme culturel et discours d'enseignants : Étude de cas d'une école primaire en Guyane ». Actes du colloque international « Éducation et territoires : contextes, organisations et trajectoires scolaires », 29 et 30 novembre 2007. Digne-les-Bains (Alpes de Haute Provence - France)

- 2008 (à paraître). « La Guyane est-elle maudite? Analyse des origines du mythe et de ses conséquences ", dans Nouvelles études francophones.

- 2008 (à paraître). « L'espace urbain cayennais : Champ de lutte identitaire », in L'ordinaire Latino-Américain, IPEALT. Université de Toulouse-Le Mirail

PIERRE-YVES CHICOT

Pierre-Yves Chicot (pierre-yves.chicot@univ-ag.fr) est Maître de conférences en droit public à l'université des Antilles et de la Guyane 